\title{
Lowering drug prices in China proves challenging
}

$\mathrm{X}$

u Kunqing's job at a food wholesaler in the bustling city of Guangzhou in southern China pays well. On top of a salary of 2000 yuan $(\$ 343)$ per month, he gets free housing and meals. But after acquiring nephritis in 2002, he developed a chronic kidney condition and the 39-year-old father of three must now spend over half of his salary on a cocktail of 10 medicines.

During his recent monthly visit to Wuzhou Red Cross Hospital near his hometown village in Guangxi province, which took three hours by bus from Guangzhou, he was charged 1216 yuan for consultations, tests and medicines.

"The price is very expensive, but I have to pay - I'm sick," he said.

What makes Xu most angry, he said, is a sense that the hospital is cheating him. Xu's medicine bill fluctuates every month, as do the prices of tests, and nothing is itemized. "I think that the doctors make money from these drugs, but I don't have any evidence." (The head of the hospital's administrative department did not respond to emailed questions.)

Within the next two years, hospitals such as Wuzhou Red Cross will almost certainly have to change. A year ago, China initiated a long-awaited pilot policy in all hospitals in Shenzhen, a city near Guangzhou, and in the Beijing Friendship Hospital, to eliminate mark-ups on medicines that have gradually inflated in price over the past three decades.

In 2008, China spent 620.24 billion yuan on pharmaceuticals, representing $42.7 \%$ of the country's total health expenditure, according to the World Bank. By comparison, Organization for Economic Co-operation and Development countries in the west, including Canada, spent on average just $17 \%$ of their health outlay on drugs in the same year.

Since China initiated economic reforms at the end of the 1970s, hospitals have increasingly had to cover their own costs as government subsidies waned. In response, medical facilities

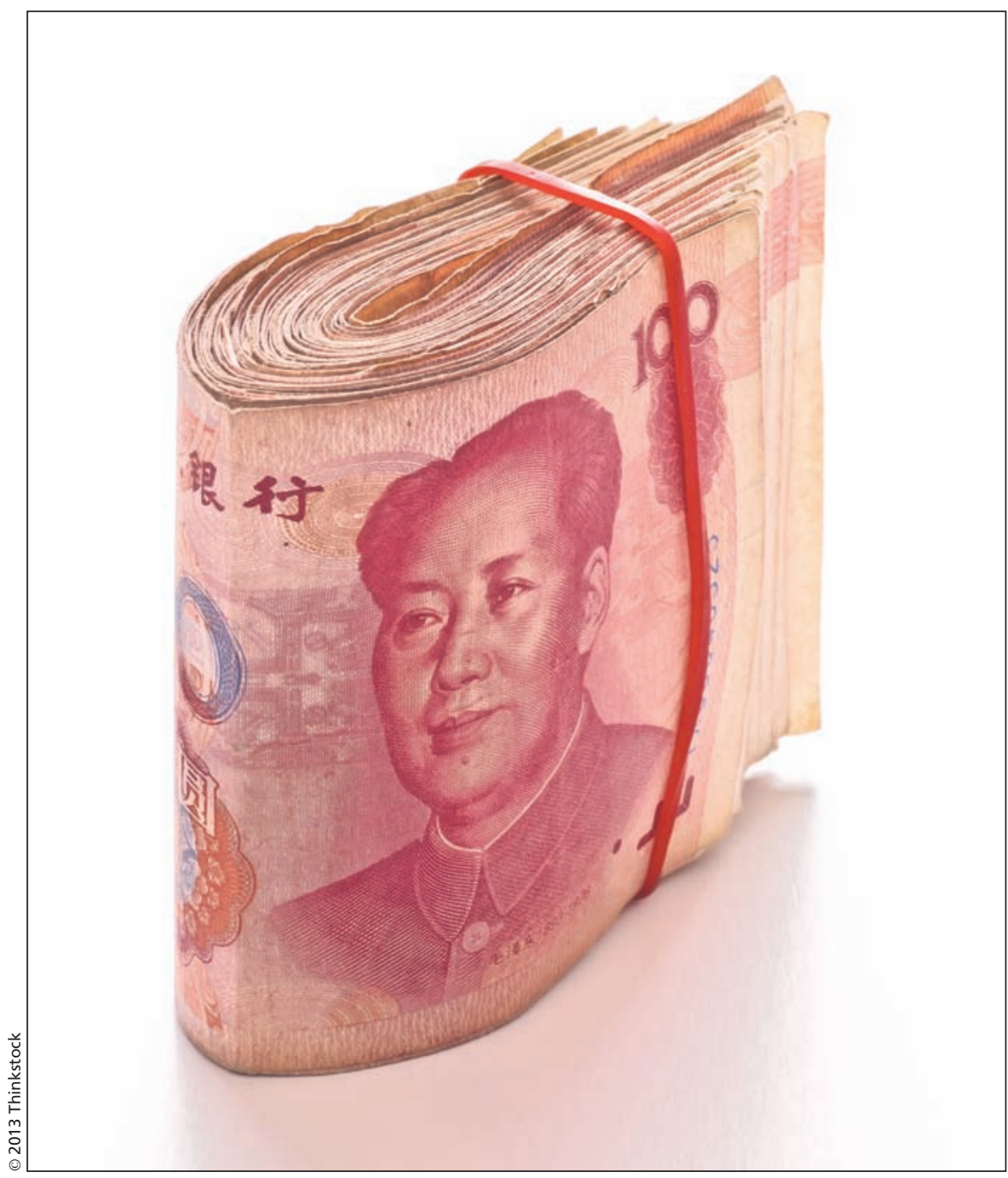

Drugs began to cost a bundle in China's hospitals in the 1970s thanks to a policy allowing medical facilities to mark up medicines to generate revenue.

across the country were permitted to mark up medicines by $15 \%$ to generate income, a policy that led to a "perverse incentive issue," said Dr. Michael O'Leary, the World Health Organization (WHO) representative in China.

"That was widely recognized as a recipe for overprescription, for overuse, for choosing medicines that are more expensive than they need to be," he added.

The pilot schemes in Shenzhen and Beijing have reversed these markups and raised government subsidies and consultation fees for nonresidents of these cities to make up the shortfall. The aim is simple: to remove the incentive to overprescribe and overprice medicines in China.

The policy was rolled out in rural clinics in 2009, though enforcing it has proved a challenge. But the real test, acknowledged by the government and the WHO, is making this approach work in China's large city hospitals, where the overheads typically run into the hundreds of millions of yuan every year.

"There are many voices and interests, and so on, in hospitals, and to take away a major source of hospital 
revenue ... remains a big challenge," said O'Leary.

In Shenzhen No. 2 People's Hospital, as part of the pilot beginning in July 2012, the pricing changes have been radical. But the public does not appear to have a good general understanding as to why medicine prices have changed in Shenzhen.

Zheng, a patient at the hospital who gave only her family name, said she had seen advertisements on local television announcing a drop in prices. She just didn't know why. Her bill for treament of menstrual discomfort came to just one yuan for the consultation the fee has now been raised from nine yuan to 21 yuan for nonresidents and about 70 yuan for medicine.

"When I paid, the price was less than expected," she said.

China is aiming to roll out and enforce these measures all across the vast country of more than 1.3 billion people within just two years. Can Beijing succeed?

Back in Wuzhou, six hours by bus from Shenzhen, just across the provincial border in poorer Guangxi province,
Mei Xuewan, branch manager of local pharmacy chain Jie Xun Medicine, is skeptical. China's powerful drug companies are an influential lobby group, she said, and hospitals are increasingly moving away from the state model towards hybrid government-private partnerships or outright private entities.

"Hospitals have to make a profit," said Mei. "Drug prices will rise continuously anyway." - Steve Finch, Shenzhen, China

CMAJ 2013. DOI:10.1503/cmaj.109-4548 